\title{
Polygones de Hodge, de Newton et de l'inertie modérée des représentations semi-stables
}

\author{
Xavier Caruso • David Savitt
}

Received: 13 June 2008 / Revised: 1 August 2008 / Published online: 2 October 2008

(C) The Author(s) 2008. This article is published with open access at Springerlink.com

\begin{abstract}
Dans cette note, on associe à chaque représentation $p$-adique semi-stable un polygone obtenu à partir des poids de l'inertie modérée de la semi-simplifiée modulo $p$, et on compare dans certains cas la position relative de ce polygone par rapport aux polygones de Hodge et de Newton.
\end{abstract}

\section{Introduction}

Soient $p$ un nombre premier, et $k$ un corps parfait de caractéristique $p$. On note $W=W(k)$ l'anneau des vecteurs de Witt à coefficients dans $k, K_{0}$ le corps des fractions de $W$ et on fixe $K$ une extension totalement ramifiée de $K_{0}$ de degré $e$, d'anneau des entiers noté $\mathcal{O}_{K}$. On fixe également $\bar{K}$ une cloture algébrique de $K$, on désigne par $G_{K}$ le groupe de Galois absolu de $K$ et par $I$ le sous-groupe d'inertie.

Si $V$ est une $\mathbb{Q}_{p}$-représentation semi-stable (voir [10]) de $G_{K}$ de dimension $d$, on sait lui associer plusieurs invariants numériques. Il y a classiquement les poids de Hodge-Tate et les pentes de l'action du Frobenius sur le module filtré de Fontaine correspondant. Ce sont tous deux des $d$-uplets de rationnels (entiers pour les poids de Hodge-Tate) que l'on représente parfois sous la forme de polygones appelés alors respectivement polygone de Hodge et polygone de Newton. (Pour les définitions de ces multiples objets, voir paragraphe 2.1.) On sait que ces deux polygones ont même point

\footnotetext{
X. Caruso $(\varangle)$

IRMAR, Université de Rennes I, Campus de Beaulieu,

35042 Rennes Cedex, France

e-mail: Xavier.caruso@normalesup.org

D. Savitt

Department of Mathematics, The University of Arizona,

617 N. Santa Rita Ave., P.O. Box 210089, Tucson, AZ 85721-0089, USA

e-mail: savitt@math.arizona.edu
} 
d'arrivée et que le polygone de Hodge est toujours situé en-dessous de celui de Newton. Si $h_{1} \leq h_{2} \leq \cdots \leq h_{d}$ sont les poids de Hodge-Tate et $n_{1} \leq n_{2} \leq \cdots \leq n_{d}$ les pentes de Frobenius, la condition visuelle sur les polygones se traduit par les inégalités

$$
h_{1}+\cdots+h_{k} \leq n_{1}+\cdots+n_{k}
$$

pour tout $k \in\{1, \ldots, d\}$ avec égalité si $k=d$.

Il existe toutefois un troisième invariant numérique que l'on peut associer à $V$. Rappelons avant de le définir que toute $\mathbb{F}_{p}$-représentation irréductible $H$ du groupe d'inertie $I$ est simplement décrite par ses poids de l'inertie modérée : ils forment une suite de $d^{\prime}=\operatorname{dim} H$ entiers compris entre 0 et $p-1$ (définie à permutation circulaire près). (Pour des précisions sur cette classification, le lecteur pourra se reporter au paragraphe 1 de [14].)

Considérons à présent $T$ un $\mathbb{Z}_{p}$-réseau dans $V$ stable par l'action du groupe de Galois (un tel réseau existe toujours par compacité de $G_{K}$ ), et focalisons-nous sur la représentation $T / p T$ restreinte au groupe d'inertie $I$. D'après les rappels que nous venons de faire, à tout quotient de Jordan-Hölder de cette représentation, il correspond une suite d'entiers compris entre 0 et $p-1$. D' autre part, un théorème de Brauer-Nesbitt (voir paragraphe 82.1 de [8]) montre que la famille des quotients de Jordan-Hölder de $T / p T$ ne dépend que de la représentation $V$ (et pas du choix de $T$ ). Ainsi, en regroupant les poids associés à chacun des quotients de Jordan-Hölder, on obtient une collection de $d$ entiers compris entre 0 et $p-1$ qui est canoniquement associée à $V$. Notons-les $i_{1} \leq i_{2} \leq \cdots \leq i_{d}$.

Il est naturel de se demander si ces entiers ont un rapport avec les autres invariants. Lorsque $e=1$ et lorsque la représentation est cristalline, Fontaine et Laffaille ont montré dans [11] que les poids de l'inertie modérée sont les mêmes que les poids de Hodge-Tate. Toutefois, ce résultat simple est mis en défaut pour les représentations semi-stables non cristallines, même dans les cas les plus simples : dimension 2 et $e=1$. Plus précisément, dans cette situation, Breuil et Mézard ont montré dans [5] par un calcul explicite que si les poids de Hodge-Tate sont 0 et $r$ avec $r<p-1$, alors les poids de l'inertie modérée peuvent être n'importe quel couple $\left(i_{1}, i_{2}\right)$ pourvu que $i_{1}+i_{2}=r$.

Dans cette note, nous dégageons des contraintes explicites sur les poids de l'inertie modérée valables pour toutes les représentations semi-stables dès lors que $e r<p-1$. Commençons pour cela par définir le polygone de l'inertie modérée comme le polygone associé aux rationnels $i_{k}^{\prime}=\frac{i_{k}}{e}$. Nous montrons le théorème suivant:

Théorème 1 Supposons que tous les poids de Hodge-Tate soient compris entre 0 et $r$ pour un entier $r$ vérifiant er $<p-1$. Alors, le polygone de l'inertie modérée est situé au-dessus du polygone de Hodge. De plus ils ont même point terminal. Autrement dit, pour tout $k \in\{1, \ldots, d\}$ :

$$
e\left(h_{1}+\cdots+h_{k}\right) \leq i_{1}+\cdots+i_{k}
$$

avec égalité si $k=d$. 
Remarque 2 L'hypothèse er $<p-1$ se justifie aisément puisque les poids de l'inertie modérée sont par définition bornés par $p-1$. On pourrait toutefois se demander si le résultat demeure avec la condition plus faible $e r \leq p-1$. La réponse est négative en général, mais il est tout à fait probable qu'elle devienne positive sous certaines hypothèses supplémentaires simples.

Forts de ce résultat, nous essayons ensuite de comprendre s'il y a un rapport entre polygone de l'inertie modérée et polygone de Newton. Au vu des exemples, la question la plus naturelle que l'on puisse se poser est la suivante:

Question 3 On suppose toujours que les poids de Hodge-Tate sont compris entre 0 et $r$ (avec er $<p-1)$. Est-il vrai que le polygone de l'inertie modérée est situé entre le polygone de Hodge et celui de Newton?

Hélas, nous n'avons pour l'instant que peu d'idées pour résoudre ce problème et nous ne savons prouver que le résultat (très) partiel suivant:

Théorème 4 Supposons que tous les poids de Hodge-Tate soient compris entre 0 et $r$ pour un entier $r$ vérifiant er $<p-1$. Alors, tant que les polygones de Hodge et de Newton ne se séparent pas, le polygone de l'inertie modérée coüncident aussi avec eux. En particulier, si polygone de Hodge et polygone de Newton coüncident, ils coüncident également avec le polygone de l'inertie modérée.

Cela se traduit encore de la façon suivante. Soit $k \in\{1, \ldots, d\}$. Supposons que pour tout $k^{\prime} \leq k$, on ait $h_{k^{\prime}}=n_{k^{\prime}}$. Alors $i_{k}=e h_{k}\left(=e n_{k}\right)$.

L'organisation de cette note se fait comme suit. La partie 2 est consacrée à des rappels sur la théorie de Breuil développée dans [1-3] et [6], qui joue un rôle central dans la démonstration des résultats précédemment annoncés. La partie 3, quant à elle, regroupe les preuves desdits résultats. Finalement, dans une dernière partie, nous donnons les résultats ${ }^{1}$ du calcul des invariants précédents sur un exemple simple en dimension 2. Il en ressort que, contrairement à ce qui se passe pour $e=1$, les polygones de Hodge et de l'inertie modérée ne sont en général pas confondus pour les représentations cristallines.

Remerciements Pendant l'accomplissement de ce travail, le second auteur a été partiellement financé par le NSF grant DMS-0600871 et lui en est reconnaissant. De plus, une partie de cet article a été écrite alors que les auteurs étaient respectivement en visite à l'Université de Bonn et au Max-Planck-Institut für Mathematik. Que ces instituts soient ici remerciés comme il se doit pour leur accueil chaleureux. C'est finalement un plaisir pour le premier auteur de remercier Christophe Breuil de lui avoir conseillé de se pencher sur la problématique de ce papier, ainsi que pour ses encouragements après les premiers résultats.

\footnotetext{
1 Afin de ne pas doubler la longueur de ce texte, le détail des calculs est réporté à un papier ultérieur [7]. Voir également la dernière version de cet article sur arXiv.org.
} 


\section{La théorie de Breuil}

Cette section est dédiée aux préliminaires de théorie de Hodge $p$-adique, rationnelle et entière. Nous rappelons dans un premier temps la correspondance, dûe à Fontaine, entre représentations $p$-adiques semi-stables et $(\varphi, N)$-modules filtrés faiblement admissibles. Ensuite, nous détaillons la théorie de Breuil qui permet de comprendre les réseaux à l'intérieur de telles représentations galoisiennes et les réductions modulo $p$ de ces réseaux.

\subsection{Théorie de Hodge $p$-adique rationnelle}

Les $(\varphi, N)$-modules filtrés de Fontaine

Définition On note $\operatorname{MF}_{K}(\varphi, N)$ la catégorie dont les objets sont la donnée de :

- un $K_{0}$-espace vectoriel $D$ de dimension finie;

- une filtration décroissante Fil ${ }^{t} D_{K}$ exhautive et séparée sur $D_{K}=D \otimes_{K_{0}} K$ par des sous $K$-espaces vectoriels;

- une application $K_{0}$-semi-linéaire (par rapport au Frobenius sur $K_{0}$ ) injective $\varphi: D \rightarrow D$ (appelée Frobenius);

- une application $K_{0}$-linéaire $N: D \rightarrow D$ (appelée monodromie) vérifiant $N \varphi=p \varphi N$.

Les morphismes dans cette catégorie sont les applications $K_{0}$-linéaires compatibles au Frobenius, à la monodromie et à la filtration après extension des scalaires à $K$. On associe à tout objet $D \in \operatorname{MF}_{K}(\varphi, N)$ deux invariants numériques qui sont son nombre de Hodge et son nombre de Newton. Si $D$ est de dimension 1, le nombre de Hodge $t_{H}(D)$ est défini comme le plus grand entier $t$ tel que Fil ${ }^{t} D_{K}=D_{K}$ alors que le nombre de Newton $t_{N}(D)$ est la pente du Frobenius sur $D$ (c'est-à-dire la valuation $p$-adique de $\alpha \in K$ tel que $\phi(x)=\alpha x$ pour un $x \in D$ non nul). Si $D$ est de dimension $d$, on pose par définition $t_{N}(D)=t_{N}\left(\Lambda^{d} D\right)$ et $t_{H}(D)=t_{H}\left(\Lambda^{d} D\right)$. Un objet $D$ est dit faiblement admissible si $t_{N}(D)=t_{H}(D)$ et si pour tout $D^{\prime} \subset D$ stable par $\varphi$ et $N$ et muni de la filtration induite, on a $t_{H}\left(D^{\prime}\right) \leq t_{N}\left(D^{\prime}\right)$. On note $\operatorname{MF}_{K}^{\mathrm{fa}}(\varphi, N)$ la sous-catégorie de $\operatorname{MF}_{K}(\varphi, N)$ formée des objets faiblement admissibles. On montre que c'est une catégorie abélienne, stable par produit tensoriel et dualité.

Polygones de Hodge et de Newton Plutôt que les nombres de Hodge et de Newton, on considère parfois les polygones de Hodge et Newton. Comme ceux-ci sont centraux dans toute cette note, nous en rappelons la définition. Rappelons dans un premier temps que si $n_{1} \leq n_{2} \leq \cdots \leq n_{d}$ sont des nombres, on leur associe un "polygone » de la façon suivante : on rejoint dans le plan les points de coordonnées $\left(k, n_{1}+\cdots+n_{k}\right)$ et on trace les verticales issues du début et de la fin de la ligne brisée précédente. Le point $\left(d, n_{1}+\cdots+n_{d}\right)$ est appelé point d'arrivée ou point terminal du polygone. $\mathrm{Si}$ les entiers $n_{i}$ ne sont pas triés, on commence par le faire.

Si maintenant $D$ est un objet de $\operatorname{MF}_{K}(\varphi, N)$, son polygone de Hodge est le polygone associé aux entiers $t$ pour lesquels $\mathrm{Fil}^{t+1} D_{K} \neq \mathrm{Fil}^{t} D_{K}$ avec la convention que l'entier 
$t$ est repété autant de fois que la dimension de $\mathrm{Fil}^{t+1} D_{K} / \mathrm{Fil}^{t} D_{K}$. Le polygone de Newton de $D$ quant à lui est le polygone associé aux pentes de l'action de Frobenius; c'est aussi le polygone de Newton (usuel) du polynôme caractéristique de la matrice de $\varphi$ dans une base quelconque de $D$ sur $K_{0}$. On montre que si $D$ est faiblement admissible alors le polygone de Hodge est en-dessous du polygone de Newton et que ceux-ci ont même point d'arrivée.

Lien avec les représentations semi-stables Dans [9], Fontaine construit un anneau $B_{\text {st }}$ muni de structures supplémentaires (dont nous ne rappelons pas la définition ici, car elle ne nous servira pas). Il montre que si $V$ est une représentation semi-stable de $G_{K}$, alors:

$$
D_{\mathrm{st}}(V)=\left(B_{\mathrm{st}} \otimes_{\mathbb{Q}_{p}} V\right)^{G_{K}}
$$

est un objet de $\operatorname{MF}_{K}^{\mathrm{fa}}(\varphi, N)$. Mieux, le foncteur $D_{\text {st }}$ établit une anti-équivalence de catégories entre la catégorie des représentations galoisiennes semi-stables et $\operatorname{MF}_{K}^{\mathrm{fa}}(\varphi, N)$. Les polygones de Hodge et de Newton de la représentation galoisienne sont alors définis comme les polygones de Hodge et de Newton de l'objet de $\operatorname{MF}_{K}^{\mathrm{fa}}(\varphi, N)$ correspondant.

\section{Les $S_{K_{0}}$-modules filtrés de Breuil}

Définitions Soit $f_{\pi}: W[u] \rightarrow \mathcal{O}_{K}$ l'application qui envoie $u$ sur $\pi$. Notons $E(u)$ le polynôme minimal de $\pi$ sur $K_{0}$ de sorte que le noyau de $f_{\pi}$ soit l'idéal principal engendré par $E(u)$. On note $S$ le complété $p$-adique de l'enveloppe à puissances divisées de $W[u]$ relativement au noyau de $f_{\pi}$ (et compatible avec les puissances divisées canoniques sur $p W[u]$ ). L'application $f_{\pi}$ se prolonge à $S$. De plus, $S$ est naturellement muni d'une filtration Fil ${ }^{i} S$ (la filtration à puissances divisées), d'un Frobenius $W$-semi-linéaire $\phi: S \rightarrow S$ continu pour la topologie $p$-adique défini par $\phi(u)=u^{p}$, et d'un opérateur de monodromie $W$-linéaire $N: S \rightarrow S$ continu pour la topologie $p$-adique, vérifiant la condition de Leibniz et défini par $N(u)=-u$. On note $c=\phi(E(u)) / p$; c'est une unité de $S$. Finalement, on désigne par $f_{0}: S \rightarrow W$ le morphisme d'anneau (continu pour la topologie $p$-adique) qui envoie $u$ et toutes ses puissances divisées sur 0 .

On pose $S_{K_{0}}=S \otimes_{W} K_{0}$; les structures supplémentaires que l'on vient de définir s'y prolongent.

Ceci permet de définir la catégorie $\mathcal{M F}(\phi, N)$. Ses objets sont la donnée de:

- un $S_{K_{0}}$-module $\mathcal{D}$ libre de rang fini;

- une filtration Fil $^{t} \mathcal{D}$ telle que Fil $^{i} S \cdot$ Fil $^{t} \mathcal{D} \subset$ Fil $^{t+i} \mathcal{D}$ pour tous $i$ et $t$;

- un Frobenius $S_{K_{0}}$-semi-linéaire $\phi: \mathcal{D} \rightarrow \mathcal{D}$;

- un opérateur de monodromie $N: \mathcal{D} \rightarrow \mathcal{D}$ vérifiant $N \phi=p \phi N$ et $N\left(\mathrm{Fil}^{t} \mathcal{D}\right) \subset$ Fil $^{t-1} \mathcal{D}$ pour tout $t$.

Une équivalence de catégories Si $D$ est un objet de $\operatorname{MF}_{K}(\varphi, N)$, on peut lui associer un objet de $\mathcal{D} \in \mathcal{M F}(\phi, N)$ de la façon suivante. On pose $\mathcal{D}=D \otimes_{K_{0}} S_{K_{0}}$. Il est 
muni de $\phi=\phi \otimes \varphi$ et de $N=N \otimes 1+1 \otimes N$. La filtration, quant à elle, est définie par:

$$
\mathrm{Fil}^{t} \mathcal{D}=\left\{x \in \mathcal{D} / \forall i \geq 0, f_{\pi}\left(N^{i}(x)\right) \in \mathrm{Fil}^{t-i} D\right\}
$$

On montre (voir [1], section 6) que cette association définit une équivalence de catégories entre $\operatorname{MF}_{K}(\varphi, N)$ et $\mathcal{M} \mathcal{F}(\phi, N)$. Un quasi-inverse est décrit comme suit. Soit $\mathcal{D} \in \mathcal{M F}(\phi, N)$. On pose $D=\mathcal{D} \otimes_{S_{K_{0}}} K_{0}$ (où le morphisme $S_{K_{0}} \rightarrow K_{0}$ est $f_{0}$ ). Il est muni des opérateurs $\phi$ et $N$ qui passent au quotient et définissent respectivement le Frobenius et l'opérateur de monodromie de l'objet de $\operatorname{MF}_{K}(\varphi, N)$. La définition de la filtration demande un peu plus de travail, et notamment un lemme préliminaire.

Lemme 5 Avec les notations précédentes, il existe une unique section $s: D \rightarrow \mathcal{D}$ de $\mathrm{id} \otimes f_{0}$, qui soit $K_{0}$-linéaire et compatible au Frobenius.

Démonstration Voir proposition 6.2.1.1 de [1].

La section $s$ du lemme fournit en particulier une flèche $K_{0}$-linéaire $D \rightarrow \mathcal{D} /$ Fil $^{1} S_{K_{0}} \mathcal{D}$. En utilisant que $\mathcal{D} / \mathrm{Fil}^{1} S_{K_{0}} \mathcal{D}$ est un espace vectoriel sur le quotient $S_{K_{0}} /$ Fil $^{1} S_{K_{0}} \simeq K$ (par la flèche $u \mapsto \pi$ ) dont le rang est le même que celui de $D$, on montre facilement que $s$ s'étend en un isomophisme $K$-linéaire $s_{K}: D_{K} \rightarrow \mathcal{D} /$ Fil $^{1} S_{K_{0}} \mathcal{D}$. La filtration est alors obtenue $\operatorname{par~Fil~}^{t} D=s_{K}^{-1}\left(\mathrm{Fil}^{t} \mathcal{D} / F i l^{1} S_{K_{0}} \mathcal{D}\right)$.

Finalement, mentionnons que de la définition de la filtration, on déduit l'égalité suivante, qui nous sera utile pour la suite:

$$
\mathrm{Fil}^{t-i} \mathcal{D}=\left\{x \in \mathcal{D} / E(u)^{i} x \in \mathrm{Fil}^{t} \mathcal{D}\right\}
$$

valable pour tous entiers $t$ et $i$.

\subsection{Théorie de Hodge $p$-adique entière et de torsion}

À partir de maintenant, on se donne un entier positif $r<p-1$ et on se restreint aux représentations pour lesquelles $\mathrm{Fil}^{0} D_{K}=D_{K}$ et Fil ${ }^{r+1} D_{K}=0$ sur le module filtré de Fontaine $D$ associé. L'intérêt d'utiliser la théorie de Breuil est qu'elle permet de décrire les réseaux dans les représentations semi-stables (vérifiant l'hypothèse précédente).

Modules fortement divisibles Soit $\mathcal{D}$ un objet de $\mathcal{M F}(\phi, N)$ associé à un objet $D \in \operatorname{MF}_{K}(\varphi, N)$ dont la filtration est comprise entre 0 et $r$. Un module fortement divisible (ou réseau fortement divisible) dans $\mathcal{D}$ est un sous- $S$-module $\mathcal{M} \subset \mathcal{D}$ vérifiant:

- $\mathcal{M}$ est libre de rang fini sur $S$;

- le morphisme naturel $\mathcal{M} \otimes_{S} S_{K_{0}} \rightarrow \mathcal{D}$ est un isomorphisme;

- $\phi\left(\mathrm{Fil}^{r} \mathcal{M}\right) \subset p^{r} \mathcal{M}$ où $\mathrm{Fil}^{r} \mathcal{M}=\mathcal{M} \cap \mathrm{Fil}^{r} \mathcal{D}$;

- $\frac{\phi}{p^{r}}\left(\mathrm{Fil}^{r} \mathcal{M}\right)$ engendre $\mathcal{M}$ sur $S$. 
On montre (voir corollaire 2.1.4 de [3]) que si $\mathcal{D}$ admet un réseau fortement divisible, alors $\mathcal{D}$ correspond à un $D$ faiblement admissible $V$, et donc à une représentation galoisienne. La réciproque est également vraie : si $e r<p-1$ (cas qui nous intéresse principalement ici), c'est le résultat principal de [3], sinon c'est une conséquence des travaux de Kisin (voir [12]) et de Liu (voir [13]). En outre, dans le cas où $D$ est faiblement admissible, la dernière condition (à savoir « $\frac{\phi}{p^{r}}\left(\operatorname{Fil}^{r} \mathcal{M}\right)$ engendre $\left.\mathcal{M} »\right)$ est impliquée par les précédentes.

D'autre part, on dispose d'un foncteur $T_{\text {st }}$ (dont on pourra trouver la définition dans [2]) qui à $\mathcal{M}$ associe un $\mathbb{Z}_{p}$-réseau stable par Galois $T$ à l'intérieur de $V$. Ce foncteur établit en fait une bijection entre les réseaux fortement divisibles dans $\mathcal{D}$ et les réseaux stables par Galois dans $V$. (Pour ce dernier résultat, voir [13].)

Les catégories $\operatorname{Mod}_{/ S_{1}}^{\phi, N}$ et $\operatorname{Mod}_{/ \tilde{S}_{1}}^{\phi, N}$ On est maintenant tenté de réduire modulo $p$ les modules fortement divisibles que l'on vient d'introduire pour obtenir une description des représentations de la forme $T / p T$. On pose pour cela $S_{1}=S / p S$. Les structures supplémentaires sur $S$ passent au quotient pour définir des structures analogues sur $S_{1}$. On introduit la catégorie $\operatorname{Mod}_{/ S_{1}}^{\phi, N}$ dont les objets sont la donnée de :

- $\quad$ un module $\mathcal{M}$ libre de type fini sur $S_{1}$;

- un sous-module $\mathrm{Fil}^{r} \mathcal{M} \subset \mathcal{M}$ contenant $\mathrm{Fil}^{r} S_{1} \mathcal{M}$;

- une application semi-linéaire $\phi_{r}: \mathrm{Fil}^{r} \mathcal{M} \rightarrow \mathcal{M}$ dont l'image engendre $\mathcal{M}$ sur $S_{1}$

- une application $N: \mathcal{M} \rightarrow \mathcal{M}$ vérifiant la condition de Leibniz, telle que $u^{e} N\left(\mathrm{Fil}^{r} \mathcal{M}\right) \subset \mathrm{Fil}^{r} \mathcal{M}$ et faisant commuter le diagramme suivant:

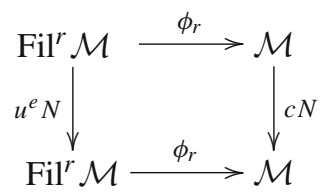

Si $e r<p-1$, la catégorie $\operatorname{Mod}_{/ S_{1}}^{\phi, N}$ est étudiée dans [6] : on montre en particulier qu'elle est abélienne et artinienne et on décrit ses objets simples lorsque le corps résiduel $k$ est algébriquement clos. De plus, elle est également équipée d'un foncteur exact $T_{\text {st }}$ vers la catégorie des $\mathbb{F}_{p}$-représentations de $G_{K}$. Finalement, si $\mathcal{M}$ est un module fortement divisible, le quotient $\mathcal{M} / p \mathcal{M}$ est un objet de $\operatorname{Mod}_{/ S_{1}}^{\phi, N}$ et si $T$ est le réseau associé à $\mathcal{M}$ par le foncteur $T_{\mathrm{st}}$, on a $T_{\mathrm{st}}(\mathcal{M} / p \mathcal{M})=T / p T$.

\section{Position relative des divers polygones}

Nous prouvons à présent les théorèmes 1 et 4 . Nous commençons par quelques rappels sur les bases adaptées qui jouent un rôle essentiel dans la démonstration du théorème 1 . 
3.1 Notion de bases adaptées

Modules libres sur les anneaux «principaux» Nous isolons ici un lemme, conséquence facile du théorème de structure des modules de type fini sur les anneaux principaux. Bien qu'élémentaire, ce lemme peut être vu comme la clé de la démonstration du résultat de comparaison entre polygone de Hodge et de l'inertie modérée.

Lemme 6 Soit A un anneau principal et $\mathfrak{p}$ un élément non nul irréductible de A. On note encore $\mathfrak{p} \subset A$ l'idéal principal maximal engendré par $\mathfrak{p}$. Soient $r<N$ et d des entiers, et $M^{\prime}$ un sous-A-module de $M=\left(A / \mathfrak{p}^{N}\right)^{d}$ tel que $\mathfrak{p}^{r} A \subset M^{\prime}$. Alors, il existe $\left(e_{1}, \ldots, e_{d}\right)$ une base de $M\left(\right.$ sur $\left.A / \mathfrak{p}^{N}\right)$ et des entiers $0 \leq n_{1} \leq n_{2} \leq \cdots n_{d} \leq r$ tels que $M^{\prime}$ soit le sous-module engendré par les $\mathfrak{p}^{n_{i}} e_{i}$.

De plus, les $n_{i}$ sont uniquement déterminés (i.e. ne dépendent pas de la base $\left.\left(e_{1}, \ldots, e_{d}\right)\right)$ et peuvent s'obtenir de la façon suivante. Soit $\left(x_{1}, \ldots, x_{D}\right)$ une famille génératrice de $M^{\prime}$. Soient $G$ la matrice $d \times D$ obtenue en écrivant en colonne les composantes des vecteurs $x_{i}$ et $\hat{G}$ une matrice à coefficients dans A relevant $G$. Alors, pour tout $k \leq d$, le nombre $n_{1}+\cdots+n_{k}$ est la plus petite valuation $\mathfrak{p}$-adique d'un mineur $k \times \bar{k}$ de $\hat{G}$.

Démonstration D'après la théorie des diviseurs élémentaires, il existe des matrices inversibles $P$ et $Q$ (à coefficients dans $A$ ) telles que :

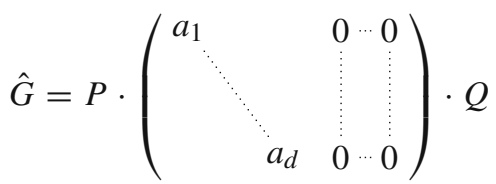

où $a_{k}$ est le PGCD des mineurs $k \times k$ de $\hat{G}$. De ceci, il découle toutes les assertions du lemme à part l'unicité des $n_{i}$. Pour cette dernière, on remarque que pour tout entier $n \leq r$ la longueur du $A$-module $\mathfrak{p}^{n} M /\left(M^{\prime} \cap \mathfrak{p}^{n} M\right)$ s'égalise avec la somme des $\max \left(0, n_{i}-n\right)$, et que la connaissance de toutes ces sommes permet de retrouver les $n_{i}$.

Existence de bases adaptées Le lemme 6 donne directement le résultat suivant, déjà bien connu.

Proposition 7 Soit $\mathcal{M}$ un module filtré sur $S_{K_{0}}$ (resp. un objet de la catégorie $\left.\operatorname{Mod}_{/ S_{1}}^{\phi, N}\right)$. Il existe $\left(e_{1}, \ldots, e_{d}\right)$ une base de $\mathcal{M}$ et des entiers $n_{1}, \ldots, n_{d}$ compris entre 0 et $r$ (resp. entre 0 et er) tels que:

$$
\begin{aligned}
\operatorname{Fil}^{r} \mathcal{M} & =\operatorname{Fil}^{p} S_{K_{0}} \mathcal{M}+\sum_{i=0}^{d} E(u)^{n_{i}} e_{i} S_{K_{0}} \\
\text { (resp. } \mathrm{Fil}^{r} \mathcal{M} & \left.=\mathrm{Fil}^{p} S_{1} \mathcal{M}+\sum_{i=0}^{d} u^{n_{i}} e_{i} S_{1}\right) .
\end{aligned}
$$

De plus les entiers $n_{i}$ sont uniquement déterminés (à l'ordre près). 
Démonstration On applique le lemme 6 respectivement aux quotients $S_{K_{0}} / \mathrm{Fil}^{p} S_{K_{0}} \simeq$ $K_{0}[u] / E(u)^{p}$ et $S_{1} / \mathrm{Fil}^{p} S_{1} \simeq k[u] / u^{e p}$.

Une base comme en fournit la proposition est appelé une base adaptée pour les entiers $n_{1}, \ldots, n_{d}$. On prendra garde au fait qu'il n'est pas vrai que tout module fortement divisible admet une base adaptée. Toutefois, c'est le cas si $r=1$ (et la démonstration repose encore sur la théorie des diviseurs élémentaires, l'anneau principal qui intervient étant ici $S / \mathrm{Fil}^{1} S \simeq \mathcal{O}_{K}$ ).

L'égalité (1) conduit directement à la proposition suivante:

Proposition 8 Soit $V$ une représentation semi-stable de $G_{K}$ et $\mathcal{D}$ son $S_{K_{0}}$-module filtré associé. Si $n_{1}, \ldots, n_{d}$ sont les entiers qui apparaissent dans l'écriture d'une base adaptée de $\mathcal{D}$, alors les poids de Hodge-Tate de $V$ sont les $h_{i}=r-n_{i}$.

\subsection{Preuve du théorème 1}

On suppose $e r<p-1$. On fixe $V$ une représentation semi-stable de $G_{K}$ de dimension $d$. On note $\mathcal{D}$ son $S_{K_{0}}$-module filtré associé. D' après le résultat principal de [3], il existe $\mathcal{M} \subset \mathcal{D}$ un réseau fortement divisible. La représentation galoisienne $T=T_{\mathrm{st}}(\mathcal{M})$ est un réseau dans $V$ stable par $G_{K}$ et on a $T / p T=T_{\text {st }}(\mathcal{M} / p \mathcal{M})$. Le but est de comparer les poids de Hodge-Tate de $V$, notés $h_{1} \leq \cdots \leq h_{d}$, avec les poids de l'inertie modérée de $T / p T$, notés $i_{1} \leq \cdots \leq i_{d}$.

Notons $n_{1} \geq \cdots \geq n_{d}$ (resp. $n_{1}^{\prime} \geq \cdots \geq n_{d}^{\prime}$ ) les entiers qui interviennent dans l'écriture d'une base adaptée de $\mathcal{D}$ (resp. de $\mathcal{M} / p \mathcal{M}$ ). Par la proposition 8 , on a la relation $h_{k}=r-n_{k}$ pour tout $k \in\{1, \ldots, d\}$. Définissons les poids de HodgeTate de $\mathcal{M} / p \mathcal{M}$ comme les rationnels $h_{k}^{\prime}=r-\frac{n_{k}^{\prime}}{e}$ et appelons polygone de Hodge de $\mathcal{M} / p \mathcal{M}$ le polygone associé à ces nombres. La démonstration se découpe alors naturellement en deux étapes : tout d'abord on montre que le polygone de Hodge de $\mathcal{M} / p \mathcal{M}$ est au-dessus de celui de $V$ (avec même point terminal), puis qu'il est au-dessous du polygone de l'inertie modérée (avec également même point terminal).

Première étape: comparaison entre polygones de Hodge On conserve les notations précédentes. Montrons tout d'abord que pour tout $k \in\{1, \ldots, d\}$, on a:

$$
e\left(n_{d}+\cdots+n_{k}\right) \leq n_{d}^{\prime}+\cdots+n_{k}^{\prime}
$$

et que l'égalité a lieu lorsque $k=1$.

Soit $\left(e_{1}, \ldots, e_{d}\right)$ une base adaptée de $\mathcal{M} / p \mathcal{M}$, donc associée aux entiers $n_{1}^{\prime}, \ldots$, $n_{d}^{\prime}$. Notons $\hat{x}_{i} \in \mathrm{Fil}^{r} \mathcal{M}$ un relevé de $u^{n_{i}^{\prime}} e_{i}$ et $x_{i}$ sa projection dans $\mathrm{Fil}^{r} \mathcal{M} / \mathrm{Fil}^{p} S \mathcal{M}$. On vérifie facilement que la famille des $x_{i}$ engendre $\mathrm{Fil}^{r} \mathcal{M} / \mathrm{Fil}^{p} S \mathcal{M}$. Soit $G$ la matrice carrée de taille $d$ obtenue en écrivant en colonne les coordonnées des vecteurs $x_{i}$ dans une base quelconque (mais fixée) de $\mathcal{M} / \mathrm{Fil}^{p} S \mathcal{M}$. C'est une matrice à coefficients dans $W[u] / E(u)^{p}$. Soient $\hat{G}$ une matrice à coefficients dans $W[u]$ relevant $G$ et $\bar{G}$ la réduction de $\hat{G}$ dans l'anneau $k[u]$.

Fixons un entier $k \in\{1, \ldots, d\}$. D'après le lemme $6, n=n_{d}+\cdots+n_{k}$ (resp. $n^{\prime}=n_{d}^{\prime}+\cdots+n_{k}^{\prime}$ ) est la plus petite valuation $E(u)$-adique (resp $u$-adique) d'un 
mineur $(d+1-k) \times(d+1-k)$ de $\hat{G}$ (resp. de $\bar{G})$. Ainsi, $E(u)^{n}$ divise certainement tous les mineurs de taile $d+1-k$ de $\hat{G}$. Par réduction modulo $p$, il s'ensuit que $u^{e n}$ divise tous les mineurs de taille $d+1-k$ de $\bar{G}$. D'où $e n \leq n^{\prime}$ comme annoncé.

Il reste à montrer que l'égalité a lieu lorsque $k=1$. Par hypothèse $\operatorname{Fil}^{r} S \mathcal{M} \subset$ Fil $^{r} \mathcal{M}$. On en déduit qu'il existe une matrice $\hat{H}$ à coefficients dans $W[u]$ telle que $\hat{G} \hat{H} \equiv E(u)^{r} I\left(\bmod E(u)^{p}\right)$ (où $I$ désigne la matrice identité). Comme $p-r \geq 1$, il existe une matrice $C$ à coefficients dans $W[u]$ telle que $\hat{G} \hat{H}=E(u)^{r}(I-E(u) C)$. En considérant les déterminants, il vient:

$$
\operatorname{det}(\hat{G}) \operatorname{det}(\hat{H})=E(u)^{r d} \Delta
$$

où $\Delta \in W[u]$ est congru à 1 modulo $E(u)$. L'anneau $W[u]$ étant factoriel, $\operatorname{det}(\hat{G})$ prend la forme $E(u)^{n} \delta$ où $n \leq r d$ est un entier et où $\delta$ divise $\Delta$. En particulier $E(u)$ ne divise pas $\delta$ et la valuation $E(u)$-adique de $\operatorname{det}(\hat{G})$ est $n$. Ainsi $n=n_{1}+\cdots+n_{d}$. D'autre part, en réduisant modulo $p$, on obtient $\operatorname{det}(\bar{G})=u^{e n} \bar{\delta}$ où $\bar{\delta}$ divise un élément congru à 1 modulo $u^{e}$. En particulier sa valuation $u$-adique est nulle et donc celle de $\operatorname{det}(\bar{G})$ vaut en. Le résultat annoncé s'ensuit.

Il résulte de ceci le résultat de comparaison, objet de ce paragraphe.

Lemme 9 Avec les notations précédentes, le polygone de Hodge de V est au-dessous du polygone de Hodge de $\mathcal{M} / p \mathcal{M}$. De plus, ils ont même point d'arrivée.

Démonstration En soustrayant $e\left(n_{1}+\cdots+n_{d}\right)=n_{1}^{\prime}+\cdots+n_{d}^{\prime}$ des deux côtés de l'inégalité précédemment prouvée, on obtient $e\left(n_{1}+\cdots+n_{k}\right) \geq n_{1}^{\prime}+\cdots+n_{k}^{\prime}$. Le résultat s'obtient alors en divisant par $(-e)$, puis en ajoutant $k r$ à cette dernière inégalité. Bien entendu, le cas d'égalité se traite par la même manipulation algébrique.

Deuxième étape: polygone de Hodge de $\mathcal{M} / p \mathcal{M}$ et polygone de l'inertie modérée Nous prouvons en fait de façon plus générale le lemme suivant:

Lemme 10 Soit $\mathcal{N}$ un objet de $\operatorname{Mod}_{/ S_{1}}^{\phi, N}$. Le polygone de Hodge de $\mathcal{N}$ est au-dessous du polygone de l'inertie modérée de $T_{\mathrm{st}}(\mathcal{N})$. De plus, ils ont même point d'arrivée.

La catégorie $\operatorname{Mod}_{/ S_{1}}^{\phi, N}$ étant abélienne et artinienne, il suffit de prouver d'une part que le résultat est vrai pour les objets simples de cette catégorie et d'autre part qu'il passe aux extensions. Comme les poids de l'inertie modérée ne dépendent que de l'action du groupe d'inertie et que les poids de Hodge-Tate sont invariants par extension non ramifiée, on peut supposer que le corps résiduel $k$ est algébriquement clos. Dans ce cas, les objets simples sont décrits dans [6] (théorème 4.3.2) et la représentation galoisienne associée à ceux-ci est également calculée dans loc. cit. (théorème 5.2.2). On constate alors sans difficulté que les polygones sont bien disposés comme on le souhaite. (En réalité, les polygones sont mêmes confondus, ici.)

Traitons à présent le cas des extensions. Donnons-nous:

$$
0 \rightarrow \mathcal{N}^{\prime} \rightarrow \mathcal{N} \rightarrow \mathcal{N}^{\prime \prime} \rightarrow 0
$$


une suite exacte dans $\operatorname{Mod}_{/ S_{1}}^{\phi, N}$. Notons $a_{1}^{\prime} \leq \cdots \leq a_{d^{\prime}}^{\prime}$ (resp. $b_{1}^{\prime} \leq \cdots \leq b_{d^{\prime}}^{\prime}$ ) les poids de Hodge-Tate de $\mathcal{N}^{\prime}$ (resp. de l'inertie modérée de $\left.T_{\text {st }}\left(\mathcal{N}^{\prime}\right)\right)$ et $a_{1}^{\prime \prime} \leq \cdots \leq$ $a_{d^{\prime \prime}}^{\prime \prime}$ (resp. $b_{1}^{\prime \prime} \leq \cdots \leq b_{d^{\prime \prime}}^{\prime \prime}$ ) ceux correspondant à $\mathcal{N}^{\prime \prime}$. Étant donné que le foncteur $T_{\text {st }}$ est exact et que les poids de l'inertie modérée de $T_{\mathrm{st}}(\mathcal{N})$ ne dépendent que des quotients de Jordan-Hölder de ladite représentation, ils sont exactement les nombres $b_{1}^{\prime}, \ldots, b_{d^{\prime}}^{\prime}, b_{1}^{\prime \prime}, \ldots, b_{d^{\prime \prime}}^{\prime \prime}$.

Évaluons maintenant les poids de Hodge-Tate de $\mathcal{N}$, que nous notons $a_{1} \leq a_{2} \leq$ $\cdots \leq a_{d}$ avec $d=d^{\prime}+d^{\prime \prime}$. D'après la preuve du lemme 6 , pour tout entier $n \leq e r$, ils vérifient la relation:

$$
\operatorname{dim}_{k} \frac{u^{n} \mathcal{N}}{u^{n} \mathcal{N} \cap \operatorname{Fil}^{r} \mathcal{N}}=\sum_{i=1}^{d} \max \left(0, e\left(r-a_{i}\right)-n\right)
$$

et l'on dispose bien entendu de formules analogues pour $\mathcal{N}^{\prime}$ et $\mathcal{N}^{\prime \prime}$. Considérons les deux applications:

$$
\frac{u^{n} \mathcal{N}^{\prime}}{u^{n} \mathcal{N}^{\prime} \cap \mathrm{Fil}^{r} \mathcal{N}^{\prime}} \rightarrow \frac{u^{n} \mathcal{N}}{u^{n} \mathcal{N} \cap \mathrm{Fil}^{r} \mathcal{N}} \rightarrow \frac{u^{n} \mathcal{N}^{\prime \prime}}{u^{n} \mathcal{N}^{\prime \prime} \cap \mathrm{Fil}^{r} \mathcal{N}^{\prime \prime}}
$$

La seconde est clairement surjective et la première est injective: en effet, si $x \in u^{n} \mathcal{N}^{\prime}$ $\mathrm{s}^{\prime}$ envoie dans $\mathrm{Fil}^{r} \mathcal{N}$, il est aussi élément de $\mathrm{Fil}^{r} \mathcal{N}^{\prime}$ en vertu de la stricte compatibilité à la filtration (voir corollaire 3.5.7 de [6]). Il s'ensuit que la dimension du terme central est supérieure à la somme des dimensions des termes extrémaux. D'où on déduit l'inégalite suivante:

$$
\begin{aligned}
\sum_{i=1}^{d} \max \left(0, e\left(r-a_{i}\right)-n\right) \geq & \sum_{i=1}^{d^{\prime}} \max \left(0, e\left(r-a_{i}^{\prime}\right)-n\right) \\
& +\sum_{i=1}^{d^{\prime \prime}} \max \left(0, e\left(r-a_{i}^{\prime \prime}\right)-n\right) .
\end{aligned}
$$

Il en résulte que pour tout $k \in\{1, \ldots, d\}$ la somme des $k$ plus petits entiers parmi les $a_{i}$ est inférieure à la somme des $k$ plus petits éléments parmi $a_{1}^{\prime}, \ldots, a_{d^{\prime}}^{\prime}, a_{1}^{\prime \prime}, \ldots, a_{d^{\prime \prime}}^{\prime \prime}$.

Par ailleurs, si $n=0$, la suite:

$$
0 \rightarrow \frac{\mathcal{N}^{\prime}}{\mathrm{Fil}^{r} \mathcal{N}^{\prime}} \rightarrow \frac{\mathcal{N}}{\operatorname{Fil}^{r} \mathcal{N}} \rightarrow \frac{\mathcal{N}^{\prime \prime}}{\mathrm{Fil}^{r} \mathcal{N}^{\prime \prime}} \rightarrow 0
$$

est exacte. En effet, il suffit d'après ce qui précède de vérifier l'exactitude au milieu. Pour cela, on considère $x \in \mathcal{N}$ qui s'envoie sur un élément $y \in \mathrm{Fil}^{r} \mathcal{N}^{\prime \prime}$ et il s'agit de montrer que $x$ s'écrit comme la somme d'un élément de $\mathcal{N}^{\prime}$ et d'un élément de $\operatorname{Fil}^{r} \mathcal{N}$. Par la stricte compatiblité à la filtration, l'application $\mathrm{Fil}^{r} \mathcal{N} \rightarrow \mathrm{Fil}^{r} \mathcal{N}^{\prime \prime}$ est surjective, d'où il existe $x^{\prime} \in \mathrm{Fil}^{r} \mathcal{N}$ qui s'envoie également sur $y \in \mathrm{Fil}^{r} \mathcal{N}^{\prime \prime}$. Mais alors $x-x^{\prime}$ est nul dans $\mathcal{N}^{\prime \prime}$ et donc est élément de $\mathcal{N}$. L'exactitude en découle. De celle-ci, on déduit que lorsque $n=0$, l'inégalité (2) est en fait une égalité, c'est-à-dire: 


$$
\sum_{i=1}^{d} a_{i}=\sum_{i=1}^{d^{\prime}} a_{i}^{\prime}+\sum_{i=1}^{d^{\prime \prime}} a_{i}^{\prime \prime}
$$

En regroupant tout ce qui précède, on s'aperçoit que l'on vient de prouver que le polygone de Hodge de $\mathcal{N}$ est situé au-dessous du polygone associé aux nombres (retriés) $a_{1}^{\prime}, \ldots a_{d^{\prime}}^{\prime}, a_{1}^{\prime \prime}, \ldots, a_{d^{\prime \prime}}^{\prime \prime}$, et qu'ils ont même point terminaux. Pour conclure, il ne reste donc plus qu'à prouver le lemme suivant.

Lemme 11 Supposons que le polygone associé aux nombres $a_{1}^{\prime}, \ldots, a_{d^{\prime}}^{\prime}$ (resp. $a_{1}^{\prime \prime}$, $\left.\ldots, a_{d^{\prime}}^{\prime \prime}\right)$ soit au-dessous du polygone associé à $b_{1}^{\prime}, \ldots, b_{d^{\prime \prime}}^{\prime}\left(\right.$ resp. $\left.b_{1}^{\prime \prime}, \ldots, b_{d^{\prime \prime}}^{\prime \prime}\right)$ et qu'ils aient même points d'arrivée.

Alors le polygone associe à $a_{1}^{\prime}, \ldots, a_{d^{\prime}}^{\prime}, a_{1}^{\prime \prime}, \ldots, a_{d^{\prime \prime}}^{\prime \prime}$ est au-dessous du polygone associé à $b_{1}^{\prime}, \ldots, b_{d^{\prime}}^{\prime}, b_{1}^{\prime \prime}, \ldots, b_{d^{\prime \prime}}^{\prime \prime}$, et ils ont même point d'arrivée.

Démonstration L'assertion sur les points d'arrivée est immédiate.

Pour le reste, on peut évidemment supposer que les $a_{i}^{\prime}$, les $a_{i}^{\prime \prime}$, les $b_{i}^{\prime}$ et les $b_{i}^{\prime \prime}$ sont rangés par ordre croissant. Soit $k$ compris entre 1 et $d$. La somme des $k$ plus petits nombres parmi $a_{1}^{\prime}, \ldots, a_{d^{\prime}}^{\prime}, a_{1}^{\prime \prime}, \ldots, a_{d^{\prime \prime}}^{\prime \prime}$ est égale à:

$$
\min _{1 \leq m \leq k}\left(a_{1}^{\prime}+\cdots+a_{m}^{\prime}\right)+\left(a_{1}^{\prime \prime}+\cdots+a_{k-m}^{\prime \prime}\right)
$$

et, bien entendu, on dispose d'une formule analogue lorsque la lettre $a$ est remplacée par $b$. La conclusion s'obtient en remarquant que chacun des termes qui apparaît dans le minimum de (3) est majoré par hypothèse par le terme correspondant où $a$ est remplacé par $b$, et que cette majoration se transporte directement sur les minimas.

\section{Remarques}

Le cas $\mathbf{e r} \geq \mathbf{p}-\mathbf{1}$ Comme nous le disions dans l'introduction, le théorème 1 peut être mis en défaut si on ôte l'hypothèse $e r<p-1$. Voici un contre-exemple très simple. Considérons $K_{0}=\mathbb{Q}_{p}$ et $K=K_{0}(\sqrt[p]{1})$; l'extension $K / K_{0}$ est totalement ramifiée de degré $e=p-1$. Le caractère cyclotomique a pour seul poids de HodgeTate 1 mais sa réduction modulo $p$ est triviale, de sorte que le poids de l'inertie modérée est 0 . On remarque qu'ici on peut choisir $r=1$, de sorte que $e r=p-1$ qui est la première situation dans laquelle l'inégalité de l'énoncé est violée.

Que dire des représentations de Hodge-Tate? Si $V$ n'est pas semi-stable, mais simplement de Hodge-Tate, l'énoncé du théorème 1 a encore un sens, et on peut légitimement se demander s'il est encore vrai dans ce contexte plus général. La réponse est négative et, là encore, les contre-exemples sont aisés à produire. On prend $K=K_{0}=\mathbb{Q}_{p}$, et on fixe $\pi \in \bar{K}$ une racine $(p-1)$-ième de $p$. Définissons le caractère $G_{K} \rightarrow \operatorname{Gal}(K(\pi) / K) \rightarrow \mathbb{Q}_{p}^{\star}, \sigma \mapsto \frac{\sigma \pi}{\pi}$. Il correspond à une représentation $p$-adique de dimension 1 qui est de Hodge-Tate ${ }^{2}$ et son seul poids de Hodge-Tate est

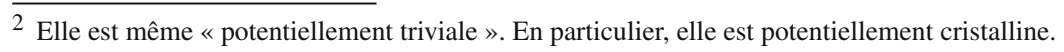


nul. Toutefois, modulo $p$, cette représentation s'identifie par construction au caractère fondamental de Serre: son poids de l'inertie modérée est donc 1 et le résultat est mis en défaut.

\subsection{Preuve du théorème 4}

On conserve notre entier positif $r$ vérifiant $e r<p-1$. De plus comme les invariants qui interviennent dans l'énoncé du théorème 4 ne changent pas après une extension non ramifiée, on peut supposer que le corps résiduel $k$ est algébriquement clos.

Soit $V$ une représentation semi-stable dont on note les poids de Hodge-Tate $h_{1} \leq$ $\cdots \leq h_{d} \leq r$ et les pentes de l'action de Frobenius $n_{1} \leq \cdots \leq n_{d}$. On suppose que les polygones de Newton et de Hodge commencent par un segment de même pente, c'est-à-dire que $h_{1}=n_{1}=s$. Notons $d^{\prime} \geq 1$ le plus grand entier pour lequel $n_{d^{\prime}}=s$. Étant donné que le polygône de Newton est situé au-dessus du polygône de Hodge, on a $n_{k}=h_{k}=s$ pour tout $k \leq d^{\prime}$.

Soit $D \in \mathrm{MF}_{K}^{\mathrm{fa}}(\varphi, N)$ le $(\varphi, N)$-module filtré de Fontaine associé à $V$. Appelons $D_{s}$ la partie de pente $s$ de $D$. D'après la définition de $d^{\prime}$, c'est un sous-espace de dimension $d^{\prime}$ stable $\varphi$. Par ailleurs l'opérateur de monodromie doit envoyer $D_{s}$ sur $D_{s-1}$ (partie de pente $s-1$ ) mais celle-ci est nulle puisque $s$ est la plus petite pente. Ainsi $N=0$ sur $D_{s}$, et $D_{s}$ est stable par $\varphi$ et $N$. Soit $D^{\prime} \subset D_{s}$ un sous-espace de dimension $n$ stable par $\varphi$ et $N$. Par les conditions de faible admissibilité, on a $t_{H}\left(D^{\prime}\right) \leq t_{N}\left(D^{\prime}\right)=n s$. Par ailleurs, Fil ${ }^{t} D_{K}=D_{K}$ pour tout $t \leq s$ et donc Fil ${ }^{t} D_{K}^{\prime}=D_{K}^{\prime}$ pour tout $t \leq s$. On en déduit qu'il n'y a pas de saut dans la filtration avant $s$ et donc que $t_{H}\left(D^{\prime}\right) \geq n s$. Finalement $t_{H}\left(D^{\prime}\right)=t_{N}\left(D^{\prime}\right)=n s$, et il s'ensuit que $D_{s}$ est un sous-objet de $D$ dans la catégorie $\operatorname{MF}_{K}^{\mathrm{fa}}(\varphi, N)$ auquel il correspond une sous-représentation $V_{s}$ de $V$.

Comme on a supposé le corps $k$ algébriquement clos, il existe une base de $D_{s}$ qui diagonalise l'action du Frobenius avec des valeurs propres toutes égales à $p^{s}$ (on rappelle que $s$ est entier puisque c'est un poids de Hodge-Tate). Il en résulte que $V_{s} \simeq \mathbb{Q}_{p}(s)^{d^{\prime}}$. Il est alors immédiat de calculer les poids de l'inertie modérée sur $V_{s}$ : on trouve qu'ils sont égaux à es comme attendu.

Les considérations précédentes démontrent le théorème 4 pour la première pente. Pour la suite, on considère le quotient $D / D_{s}$ auquel on réapplique les arguments précédents. On continue comme cela jusqu'à ce que les polygones se séparent, et cela clôt la preuve du théorème.

Remarques Comme nous le disions déjà dans l'introduction, le résultat précédent n'est pas réellement satisfaisant, et on aimerait plutôt disposer d'une comparaison entre polygone de l'inertie modérée et polygone de Newton qui ne repose sur aucune hypothèse (voir question 3 de l'introduction). On peut également s'interroger sur la position relative du polygone de Newton et celui de Hodge de la réduction modulo $p$ :

Question 12 On suppose $r<p-1$. Soit $\mathcal{M}$ un module fortement divisible. Est-il vrai que le polygone de Hodge de $\mathcal{M} / p \mathcal{M}$ est situé dessous le polygone de Newton de $D=\mathcal{M} \otimes_{S} K_{0}$ ? 
L'avantage de la question 12 (par rapport à celle de l'introduction) est qu'elle peut se poser pour tout $r<p-1$ : on entend par là qu'elle n'est semble-t-il pas trivialement mise en défaut lorsque $e r \geq p-1$ comme c'est le cas pour la question 3 . En revanche, elle propose un résultat un peu plus faible. Hélas, on dispose de trop peu d'exemples pour se faire une idée claire de la réponse aux questions précédentes. Lorsque $e=1$ (et $r<p-1$ ), Breuil et Mézard ont complètement classifié dans [5], les représentations semi-stables de dimension 2 et ont calculé les poids de l'inertie modérée pour chacune d'entre elles. Leurs résultats sont résumés dans la partie 5 de [4], et montrent que la réponse à la question 3 (et donc aussi à la question 12) est affirmative dans ce cas. Toutefois, cela ne doit pas être pris comme un indicateur très fort car dans la situation étudiée ici, soit la représentation est cristalline et alors le polygone de l'inertie modérée s'identifie avec le polygone de Hodge par les résultats de Fontaine-Laffaille, soit la représentation n'est pas cristalline, et alors le polygone de Newton est « le plus haut possible».

\section{Sans l'opérateur de monodromie}

La constatation initiale est la suivante: les objets polygone de Hodge, polygone de Hodge de la réduction modulo $p$, polygone de Newton et polygone de l'inertie modérée se calculent directement sur les modules de la théorie de Breuil, indépendamment des représentations. Mieux encore, leur calcul ne fait jamais intervenir l'opérateur de monodromie $N$. Précisément, on a vu que le polygone de Hodge et le polygone de Hodge de la réduction modulo $p$ s'obtiennent à partir des entiers qui interviennent dans l'écriture d'une base adaptée des modules correspondants. Le polygone de Newton d'un $S_{K_{0}}$-module filtré $\mathcal{D}$ est construit à partir des pentes de l'action du Frobenius sur $\mathcal{D} \otimes_{S_{K_{0}}} K_{0}$. Finalement, le polygone de l'inertie modérée de $\mathcal{M}$ peut s'obtenir en recollant les polygones de Hodge des quotients de Jordan-Hölder ${ }^{3}$ de $\mathcal{M} / p \mathcal{M}$, ce dernier n'était défini que pour $e r<p-1$.

Par ailleurs, on s'aperçoit que dans les énoncés des théorèmes 1 et 4 , il revenait au même de débuter avec un module fortement divisible plutôt qu'une représentation semi-stable. En effet, si l'on part d'une représentation semi-stable, on peut trouver un module fortement divisible dans son $S_{K_{0}}$-module filtré associé comme cela a déjà été expliqué. Et réciproquement, si l'on part d'un module fortement divisible, le $S_{K_{0}}$ module filtré obtenu en inversant $p$ correspond à un module filtré de Fontaine qui est faiblement admissible (corollaire 2.1.4 de [3]), et donc à une représentation galoisienne semi-stable.

Au vu de ces remarques, il est légitime de se demander si les théorèmes 1 et 4 s'étendent à une situation plus générale où l'on débuterait avec un pseudo-module fortement divisible (c'est-à-dire un module fortement divisible sans l'opérateur $N$ ).

\footnotetext{
3 On peut montrer (voir [6]) qu'il existe une sous-catégorie commune à $\operatorname{Mod}_{/ S_{1}}^{\phi, N}$ à $\operatorname{Mod}_{/ S_{1}}^{\phi}$ (même définition que $\operatorname{Mod}_{/ S_{1}}^{\phi, N}$ mais sans le $N$ ) qui contient tous les objects simples de ces deux catégories. Ainsi, avec la définition que l'on donne, le polygone de l'inertie modérée est le même qu'il soit calculé dans $\operatorname{Mod}_{/ S_{1}}^{\phi, N}$ ou $\operatorname{Mod}_{/ S_{1}}^{\phi}$.
} 
Pour le théorème 1 , la réponse est affirmative et la démonstration est textuellement la même que celle que nous avons déjà donnée puisqu'elle ne fait aucunément intervenir l'opérateur de monodromie. Les auteurs, par contre, ne savent pas ce qu'il en est pour le théorème 4. Toutefois, il est possible de produire un contre-exemple à la généralisation évidente de la question 3 à cette situation.

On suppose que $r=2 n$ est un entier pair, que $e=1$ et que l'uniformisante choisie est $p$ de sorte que $E(u)=u-p$. Soit le pseudo-module fortement divisible défini par $\mathcal{M}=S e_{1} \oplus S e_{2}$. On le munit de $\mathrm{Fil}^{r} \mathcal{M}$ engendré par $(u-p)^{n} e_{1}, p e_{1}+(u-p)^{n} e_{2}$ et Fil $^{p} S \mathcal{M}$, et du Frobenius $\phi$ défini $\operatorname{par} \phi\left(e_{1}\right)=c^{n} p^{n} e_{2}$ et $\phi\left(e_{2}\right)=c^{n} p^{n} e_{1}-p e_{2}$. Les entiers qui apparaissent dans l'écriture d'une base adaptée de $\mathcal{M} / p \mathcal{M}$ se calculent directement par le lemme 6 et valent $n$ et $n$. Le polygone de Hodge de $\mathcal{M} / p \mathcal{M}$ a donc pour seule $n$, et comme le polygone de l'inertie modérée doit être situé au-dessus, il a lui aussi pour seule pente $n$. Cependant, on calcule facilement les pentes du Frobenius sur $\mathcal{M} \otimes_{S} K_{0}$ : elles valent 1 et $r-1$. Le polygone de Newton est donc strictement en-dessous de celui de l'inertie modérée.

\section{Un (contre-)exemple dans le cas cristallin}

Comme nous l'avons dit dans l'introduction, les travaux de Fontaine et Laffaille assurent que dans le cas cristallin (i.e. $N=0$ sur le $(\varphi, N)$-module filtré de Fontaine) non ramifié (i.e. $e=1$ ), le polygone de l'inertie modérée s'identifie avec celui de Hodge. Nous avons également déjà dit que Breuil et Mézard ont montré par un calcul explicite que ce résultat simple est mis en défaut dès que la représentation n'est plus supposée cristalline (même dans le cas $e=1$ ). Dans cette dernière section, nous présentons un exemple duquel il découle en particulier que l'identification entre polygone de l'inertie modérée et polygone de Hodge n'est pas non plus satisfaite en général pour les représentations cristallines dès que $e \geq 2$ (voir théorème 13 et la remarque qui le suit). Le cas de Fontaine-Laffaille apparaît donc, de ce point de vue, comme très isolé. Nous ne donnons ici que les résultats et renvoyons à [7] pour le détail des calculs (on pourra également consulter la dernière version de cet article sur arXiv.org).

Pour $\mathcal{L} \in K$, on considère le $(\varphi, N)$-module filtré suivant:

$$
\left\{\begin{array}{l}
D(\mathcal{L})=K_{0} e_{1} \oplus K_{0} e_{2} \\
\varphi\left(e_{1}\right)=p e_{1}, \varphi\left(e_{2}\right)=p e_{2}, N=0 \\
\mathrm{Fil}^{0} D(\mathcal{L})_{K}=D(\mathcal{L})_{K}, \mathrm{Fil}^{3} D(\mathcal{L})_{K}=0 \\
\mathrm{Fil}^{1} D(\mathcal{L})_{K}=\mathrm{Fil}^{2} D(\mathcal{L})_{K}=K\left(\mathcal{L} e_{1}+e_{2}\right)
\end{array}\right.
$$

Il est admissible si, et seulement si $\mathcal{L} \notin \mathbb{Q}_{p}$, et on supposera toujours dans la suite que c'est le cas. Le polygone de Hodge (resp. de Newton) de $D(\mathcal{L})$ est directement visible sur la description précédente: c'est celui qui a pour pentes 0 et 2 (resp. 1 et 1). Le polygône de l'inertie modérée, quant à lui, dépend de la valeur de $\mathcal{L}$, comme nous allons le voir immédiatement. Écrivons pour cela $\mathcal{L}=a+p^{n} \mathcal{L}_{0}$ où $a \in \mathbb{Q}_{p}$ et $\mathcal{L}_{0}$ vérifie l'une des deux hypothèses suivantes (on rappelle que $\mathcal{L}$ est supposé ne pas appartenir à $\mathbb{Q}_{p}$ ): 
(i) $v_{p}\left(\mathcal{L}_{0}\right)=0$ et l'image de $\mathcal{L}_{0}$ dans le corps résiduel n'appartient pas au souscorps premier

(ii) $0<v_{p}\left(\mathcal{L}_{0}\right)<1$.

où $v_{p}$ désigne la valuation $p$-adique normalisée par $v_{p}(p)=1$.

Définissons à présent $L_{0} \in K_{0}[u]$ comme l'unique polynôme de degré $<e$ tel que $L_{0}(\pi)=\mathcal{L}_{0}$. Comme l'on a supposé $0 \leq v_{p}(\mathcal{L})<1, L_{0}$ est à coefficients dans $W$ et nous appelons $\lambda$ son terme constant. La quantité qui intervient de façon essentielle dans le calcul du polygône de l'inertie modérée est la suivante :

$$
t(\pi)=\frac{p L_{0}^{\prime}(\pi)}{E^{\prime}(\pi)\left(\phi(\lambda)-\mathcal{L}_{0}\right)}
$$

où $L_{0}^{\prime}$ et $E^{\prime}$ sont respectivement les polynomes dérivés de $L_{0}$ et $E$. On peut montrer que $t(\pi)$ appartient à $\mathcal{O}_{K}$.

Théorème 13 Le polygone de Hodge de $\overline{\mathcal{M}}$ a pour pentes $v_{p}\left(\mathcal{L}_{0}\right)$ et $2-v_{p}\left(\mathcal{L}_{0}\right)$. De plus, si $v=v_{p}(t(\pi))$, on $a$

1. si $0 \leq v<1$, alors $T / p T$ est réductible et les pentes de son polygone de l'inertie modérée sont $1-v$ et $1+v$;

2. si $v \geq 1$, alors $T / p T$ est irréductible et les pentes de son polygone de l'inertie modérée sont 0 et 2 (i.e. le polygone de l'inertie modérée est confondu avec le polygône de Hodge).

\section{Démonstration Voir [7].}

Lorsque $e=1$, on vérifie directement que $t(\pi)=0$, et donc, d'après le théorème, $T / p T$ est toujours irréductible et son polygone de l'inertie modérée a pour pentes 0 et 2 . $\mathrm{Si}$, au contraire, $e>1$, alors tous les couples $\left(i_{1}, i_{2}\right)$ d'éléments de $\frac{1}{e} \mathbb{N}$ vérifiant $i_{1} \leq i_{2}$ et $i_{1}+i_{2}=2$ peuvent apparaître comme pentes du polygone de l'inertie modérée. En effet, si $x$ est n'importe quel élément de $\mathcal{O}_{K_{0}}^{\times}$dont la réduction modulo $p$ n'appartient pas au sous-corps premier, et si $0<j<e$ est un entier, alors les paramètres $\mathcal{L}=\pi^{j}, x+\pi^{j}$ et $x$ conduisent respectivement à $v=0, \frac{j}{e}$ et $\infty$.

Open Access This article is distributed under the terms of the Creative Commons Attribution Noncommercial License which permits any noncommercial use, distribution, and reproduction in any medium, provided the original author(s) and source are credited.

\section{References}

1. Breuil, C.: Représentations $p$-adiques semi-stables et transversalité de Griffiths. Math. Annalen 307, 191-224 (1997)

2. Breuil, C.: Construction de représentations p-adiques semi-stables. Ann. Scient. ENS. 31, 281-327 (1997)

3. Breuil, C.: Représentation semi-stables et modules fortement divisibles. Invent. Math. 136, 89-122 (1999)

4. Breuil, C.: Integral p-adic Hodge theory. Adv. Stud. Pure Math. 36, 51-80 (2002)

5. Breuil, C., Mézard, A.: Multiplicités modulaires et représentations de $\mathrm{GL}_{2}\left(\mathbb{Z}_{p}\right)$ et de $\operatorname{Gal}\left(\overline{\mathbb{Q}}_{p} / \mathbb{Q}_{p}\right)$ en $\ell=p$. Duke Math. J. 115, 205-310 (2002) 
6. Caruso, X.: Représentations semi-stables de torsion dans le cas $e r<p-1$. J. Reine Angew. Math. 594, 35-92 (2006)

7. Caruso, X., Savitt, D.: Poids de l'inertie modérée de certaines représentations cristallines, en préparation

8. Curtis, C., Reiner, I.: Representation theory of finite groups and associative algebras. Interscience Publ., New York (1962)

9. Fontaine, J.M.: Le corps des périodes $p$-adiques, Astérisque 223, Soc. math. France (1994), 59-111

10. Fontaine, J.M.: Représentations $p$-adiques semi-stables, Astérisque 223, Soc. math. France (1994), 113-184

11. Fontaine, J.M., Laffaille, G.: Construction de représentations p-adiques. Ann. Scient. ENS. 15, 547-608 (1982)

12. Kisin, M.: Crystalline representations and F-crystals, Algebraic Geometry and Number Theory, Drinfeld 50th Birthday volume, 459-496

13. Liu, T.: On lattices in semi-stable representations: a proof of a conjecture of Breuil. Compositio Math. 144, 61-88 (2008)

14. Serre, J.P.: Propriétés galoisiennes des points d'ordre fini des courbes elliptiques. Invent. Math. 15, 259331 (1972) 